\title{
The methane ice rich surface of large TNO 2005 FY: a Pluto-twin in the trans-neptunian belt?
}

\author{
J. Licandro ${ }^{1,2}$, N. Pinilla-Alonso ${ }^{3}$, M. Pedani ${ }^{3}$, E. Oliva ${ }^{3,4}$, G. P. Tozzi ${ }^{4}$, and W. M. Grundy ${ }^{5}$ \\ 1 Isaac Newton Group, PO Box 321, 38700, Santa Cruz de La Palma, Tenerife, Spain \\ e-mail: licandro@ing.iac.es \\ 2 Instituto de Astrofísica de Canarias, c/Vía Láctea s/n, 38205, La Laguna, Tenerife, Spain \\ ${ }^{3}$ Fundación Galileo Galilei \& Telescopio Nazionale Galileo, PO Box 565, 38700, S/C de La Palma, Tenerife, Spain \\ ${ }^{4}$ INAF - Osservatorio Astrofisico di Arcetri, Largo e Fermi 5, 50125, Firenze, Italy \\ 5 Lowell Observatory, 1400 West Mars Hill Road, Flagstaff, AZ 86001-4470, USA
}

Received 13 October 2005 / Accepted 21 November 2005

\section{ABSTRACT}

Context. The population of known large trans-neptunian objects (TNOs) is growing very fast and the knowledge of their physical properties is a key issue to understand the origin and evolution of the Solar System.

Aims. In this paper we studied the surface composition of the recently discovered TNO $2005 \mathrm{FY}_{9}$, one of the largest known TNOs ( $\sim 0.7$ times the diameter of Pluto, i.e. $1600 \mathrm{~km}$, if the albedo is similar, or $3100-1550 \mathrm{~km}$ in diameter assuming an albedo range $0.2<p_{V}<0.8$ ).

Methods. We report visible and near infrared spectra covering the 0.35-2.5 $\mu \mathrm{m}$ spectral range, obtained with the $4.2 \mathrm{~m}$ William Herschel Telescope and the Italian $3.58 \mathrm{~m}$ Telescopio Nazionale Galileo at "El Roque de los Muchachos" Observatory (La Palma, Spain).

Results. The spectrum of this large TNO is similar to that of Pluto, with an infrared region dominated by very prominent absorptions bands formed in solid $\mathrm{CH}_{4}$. At wavelengths shorter than $0.6 \mu \mathrm{m}$, the spectrum is almost featureless and red. The red color most likely indicates the presence of complex organics, as has been hypothesized for Pluto and many other TNOs. The icy- $\mathrm{CH}_{4}$ bands in this new giant $\mathrm{TNO}$ are significantly stronger than those of Pluto, implying that methane could be even more abundant on its surface. The existence of a volatile such as methane on the surface of $2005 \mathrm{FY}_{9}$, likely accompanied by $\mathrm{N}_{2}$ and CO ices, coupled with its large size, make this Pluto-like TNO an excellent candidate to have an atmosphere comparable to Pluto's.

Key words. minor planets - comets - infrared - trans-neptunian objects

\section{Introduction}

Identified as the source of the short period comets by Fernández (1980), the trans-neptunian region is populated by icy bodies (TNOs), remnant planetesimals from the early solar system formation stages (Edgeworth 1949; Kuiper 1951). They are probably the most pristine objects in the Solar System. Temperatures in this region $(\sim 40 \mathrm{~K})$, are low, so ices trapped at formation should be preserved and can provide key information on the composition and early conditions of the pre-solar nebula. Until recently, only water ice was clearly detected in the spectra of several TNOs, e.g. 1996 TO$_{66}$ (Brown et al. 1999), (20000) Varuna (Licandro et al. 2001), (50000) Quaoar (Jewitt \& Luu 2004; Pinilla et al. 2004), (90482) Orcus (Fornasier et al. 2004), $2002 \mathrm{TX}_{300}$ (Licandro et al. 2005). On the other hand, the spectrum of the most prominent member of the trans-neptunian belt, Pluto, is dominated by strong methane ice absorption bands and weak but unambiguous signatures of $\mathrm{CO}$ and $\mathrm{N}_{2}$-ice (e.g.
Cruikshank 1998). These bands are also detected in the spectrum of Neptune's satellite Triton (Cruikshank et al. 1993), a possibly captured ex-TNO.

The recent discovery of three very bright TNOs, 2003 EL $_{61}$ (Ortiz et al. 2005), $2003 \mathrm{UB}_{313}$ and $2005 \mathrm{FY}_{9}$ (Brown et al. 2005a, 2005b) with $V \sim 17.5, V \sim 18.9$ and $V \sim 16.9$ respectively, provide an excellent opportunity to obtain spectra of TNOs with $\operatorname{good} \mathrm{S} / \mathrm{N}$.

TNO $2005 \mathrm{FY}_{9}$ is one of the largest and brightest known objects in the trans-neptunian belt according to its absolute magnitude $\left(H_{V} \sim-0.1\right)$. If the surface albedo is similar to that of Pluto, as suggested by the similarity of the spectra presented in this paper, its diameter is about 0.7 times that of Pluto $(2350 \mathrm{~km})$, i.e. $\sim 1600 \mathrm{~km}$, larger than Charon $(1250 \mathrm{~km})$. In this paper we present visible and near-infrared spectroscopy of $2005 \mathrm{FY}_{9}$ and derive mineralogical information from its surface. 


\section{Observations}

We observed $2005 \mathrm{FY}_{9}$ on 2005 August 1.87 UT simultaneously with two telescopes at the "Roque de los Muchachos Observatory" (ORM, Canary Islands, Spain), namely the $4.2 \mathrm{~m}$ William Herschel (WHT) and the Italian $3.6 \mathrm{~m}$ Telescopio Nazionale Galileo (TNG), under photometric conditions. The TNO had heliocentric distance 51.89 AU, geocentric distance 52.56 AU and phase angle $0.8^{\circ}$.

The visible spectrum $(0.35-0.98 \mu \mathrm{m})$ was obtained using the low resolution gratings (R300B in the blue arm, with a dispersion of $0.86 \AA$ /pixel, and the R158R with a dispersion of $1.63 \AA$ /pixel) of the spectrograph ISIS at WHT, and a 5" slit width oriented at the parallactic angle to minimize the spectral effects of atmospheric dispersion. The tracking was at the TNO proper motion. Four $300 \mathrm{~s}$ spectra were obtained by shifting the object by $10^{\prime \prime}$ in the slit to better correct the fringing. Calibration and extraction of the spectra were done using IRAF and following standard procedures (Massey et al. 1992). The four spectra of the TNO were averaged. The reflectance spectrum was obtained by dividing the spectrum of the TNO by the spectrum of the solar-analogue star BS4486 obtained the same night at a similar airmass just before the TNO spectrum.

The near-infrared spectrum was obtained using the high throughput, low resolution spectroscopic mode of the NearInfrared Camera and Spectrometer at the TNG, NICS, with an Amici prism disperser. This mode yields a complete $0.8-2.5 \mu \mathrm{m}$ spectrum. We used a $1.5^{\prime \prime}$ wide slit corresponding to a spectral resolving power $R \sim 34$ along the spectrum. The slit was oriented at the parallactic angle and the tracking was at the TNO proper motion. We used the observing and reduction procedure described by Licandro et al. (2002). The total exposure time is $1080 \mathrm{~s}$. To correct for telluric absorption and to obtain the relative reflectance, the $\mathrm{G}$ star Landolt 107-998 (Landolt 1992) was observed just before and after the TNO and was used as a solar analogue. Finally, the near-infrared spectrum was scaled to match the visible spectrum in the $0.80-0.98 \mu \mathrm{m}$ region. Considering that the seeing was $0.8^{\prime \prime}$, the final resolving power of the spectrum is $\sim 1000-1200$ in the visible and $\sim 60$ in the $1-2.5 \mu \mathrm{m}$ region.

The final VNIR reflectance spectrum, normalized at $0.6 \mu \mathrm{m}$ is plotted in Fig. 1. The spectra of Pluto and pure methane ice are also plotted for comparison.

\section{Discussion}

The similarity between the spectra of Pluto and $2005 \mathrm{FY}_{9}$ is striking. All the very prominent absorption bands observed in Pluto's spectrum, which correspond to $\mathrm{CH}_{4}$ ice bands, are also visible in that of $2005 \mathrm{FY}_{9}$. In particular in the visible region (see Fig. 2), almost all the methane ice absorption bands reported by Grundy et al. (2002), even the weaker ones, are detected (see Table 1) and are much deeper than those in Pluto's spectrum. The prominent bands at $0.73 \mu \mathrm{m}$ and $0.89 \mu \mathrm{m}$ are $\sim 6$ and $\sim 3$ times deeper respectively, while bands in the near infrared spectrum are $<2$ times deeper, though it is more difficult to compare at those wavelengths, where our spectral resolution is limited.

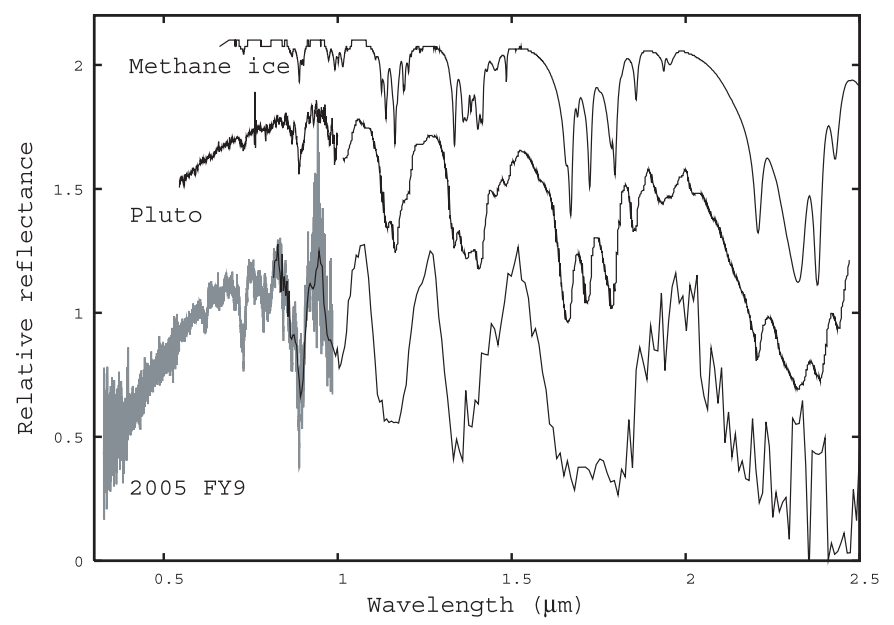

Fig. 1. Visible (grey) and near-infrared (black) reflectance spectra of $2005 \mathrm{FY}_{9}$ obtained on August 1st, 2005. The spectrum of Pluto (Grundy \& Fink 1996), normalized at $0.6 \mu \mathrm{m}$ and the spectrum of pure methane ice, both shifted vertically, are plotted for comparison. The spectrum of $2005 \mathrm{FY}_{9}$ is very similar to that of Pluto and reveals important features: (1) the slope of the continuum in the visible range is red, indicative of the presence of complex organics; (2) there are several $\mathrm{CH}_{4}$ ice absorption bands; (3) The methane ice absorption bands in the spectrum of $2005 \mathrm{FY}_{9}$ are deeper than the same bands in Pluto's spectrum.

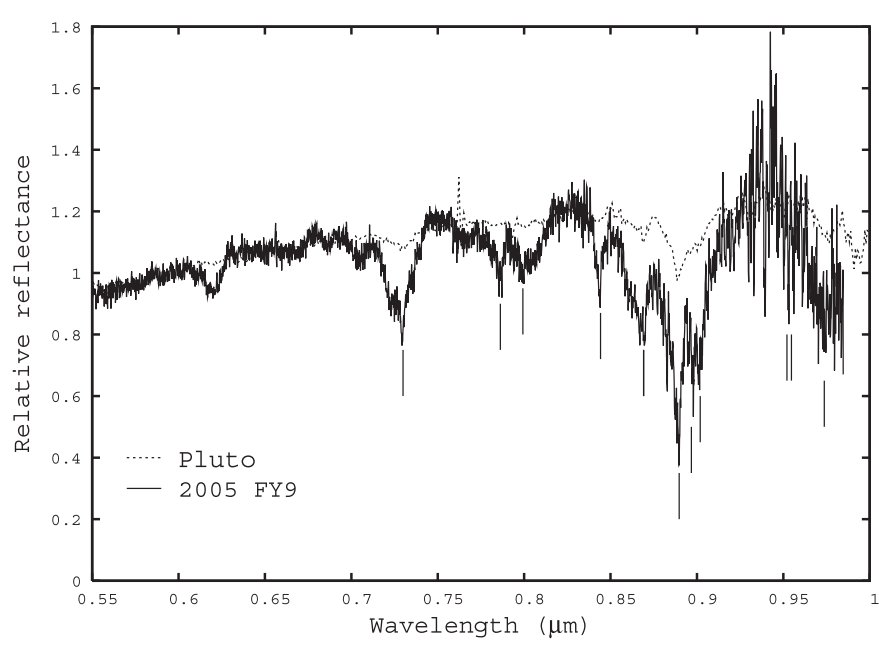

Fig. 2. Reflectance spectrum of $2005 \mathrm{FY}_{9}$ in the visible range compared to a reflectance spectrum of Pluto (Grundy \& Fink 1996; Brown $\&$ Calvin 2000), both normalized at $06 \mu \mathrm{m}$. Vertical marks indicate the central position of pure methane ice bands (Grundy et al. 2002). (1) the slope of the continuum in the visible range is red, indicative of the presence of complex organics; (2) there are numerous absorptions bands of $\mathrm{CH}_{4}$ ice; (3) the methane ice absorption bands in the spectrum of $2005 \mathrm{FY}_{9}$ are deeper than the same bands in Pluto's spectrum, at least at shorter wavelengths.

Although uncertainty regarding the absolute albedo of $2005 \mathrm{FY}_{9}$ makes quantitative spectral modeling premature, we can already conclude from its deeper $\mathrm{CH}_{4}$ bands that light reflected from $2005 \mathrm{FY}_{9}$ samples larger mean optical path lengths in $\mathrm{CH}_{4}$ ice than light from Pluto does. Larger grain sizes would accomplish this, as would higher $\mathrm{CH}_{4}$ concentrations dissolved in nitrogen ice. Broader geographic distribution of $\mathrm{CH}_{4}$ ice on 
Table 1. Position of methane lines. Laboratory data from Grundy et al. (2002), Pluto data (with uncertainties $\sim 10 \AA$ ) from Grundy \& Fink (1996).

\begin{tabular}{cccc}
\hline \hline Band & Pure methane $(\AA)$ & $2005 \mathrm{FY}_{9}(\AA)$ & Pluto $(\AA)$ \\
\hline $3 v_{1}+4 v_{4}$ & 7299 & 7296 & 7290 \\
$3 v_{3}+3 v_{4}$ & 7862 & 7860 & - \\
$3 v_{1}+3 v_{4}$ & 7993 & 7888 & 8015 \\
$v_{1}+3 v_{3}$ & 8442 & 8437 & 8430 \\
$3 v_{3}+2 v_{4}$ & 8691 & 8685 & 8690 \\
$2 v_{1}+v_{3}+2 v_{4}$ & 8897 & 8891 & 8885 \\
$2 v_{3}+4 v_{4}$ & 9019 & 9015 & - \\
\hline
\end{tabular}

$2005 \mathrm{FY}_{9}$ could contribute as well, since Pluto's $\mathrm{CH}_{4}$ ice is inhomogeneously distributed (Grundy \& Buie 2001). We note that the weaker $\mathrm{CH}_{4}$ bands at shorter wavelengths require especially large path lengths in $\mathrm{CH}_{4}$ ice, since absorption by those bands is much weaker than the stronger, near-infrared bands, which require relatively little $\mathrm{CH}_{4}$ to produce deep absorption bands. Consequently, the shorter wavelengths are particularly sensitive to regions having the most abundant $\mathrm{CH}_{4}$ ice.

The other important characteristic of the spectrum is its colour in the visible region. The red colour is indicative of the presence of a strong ultraviolet absorber. To compare with Pluto we computed the ratio of the reflectance spectrum at 0.825 and $0.590 \mu \mathrm{m}$ as in Grundy \& Fink (1996). The value of this ratio is 1.21 , almost equal to that Pluto (1.20). This corresponds to a spectral slope $S^{\prime}=8.9 \% / 1000 \AA$, a red colour typical of TNOs. The most accepted hypothesis is that such a red colour is due to complex organics molecules (tholins) formed from simple organics by photolysis (e.g. Khare et al. 1984).

While Pluto's spectrum is dominated by the strong $\mathrm{CH}_{4}$ absorption bands first observed by Cruikshank et al. (1976), two other volatile ices have since been indisputably detected: $\mathrm{CO}$ and $\mathrm{N}_{2}$ (Owen et al. 1993). The hexagonal $\beta$ phase of $\mathrm{N}_{2}$ ice was detected by means of its $2.15 \mu \mathrm{m}$ absorption band and $\mathrm{CO}$ ice was detected by means of a pair of narrow bands at 2.35 and $1.58 \mu \mathrm{m}$. Our spectral resolution is much too low to see the $\mathrm{CO}$ absorptions. The $\mathrm{N}_{2}$ band would also be difficult to detect, even if $\mathrm{N}_{2}$ were a major component of the surface of $2005 \mathrm{FY}_{9}$, because the nitrogen absorption has about a factor of a thousand smaller peak absorption coefficient than that of the nearby $\mathrm{CH}_{4}$ band at $2.2 \mu \mathrm{m}$, which dominates that spectral region. We looked for absorptions of solid $\mathrm{CO}$ and $\mathrm{N}_{2}$ in the spectrum of $2005 \mathrm{FY}_{9}$ but, unfortunately, the resolution and $\mathrm{S} / \mathrm{N}$ of our near-infrared spectrum are insufficient to say anything about the absorption bands of either species. It is also possible that surface temperatures on $2005 \mathrm{FY}_{9}$ might be below the $36.5 \mathrm{~K}$ transition temperature between the warmer $\beta$ phase of $\mathrm{N}_{2}$ ice, and the colder, cubic $\alpha$ phase of $\mathrm{N}_{2}$ ice, which has an extremely narrow $2.15 \mu \mathrm{m}$ absorption, which would be unresolved in our data (e.g., Grundy et al. 1993). Future, higher spectral resolution observations will put more constraints on the presence of $\mathrm{N}_{2}$ and $\mathrm{CO}$ ice in the surface of $2005 \mathrm{FY}_{9}$.

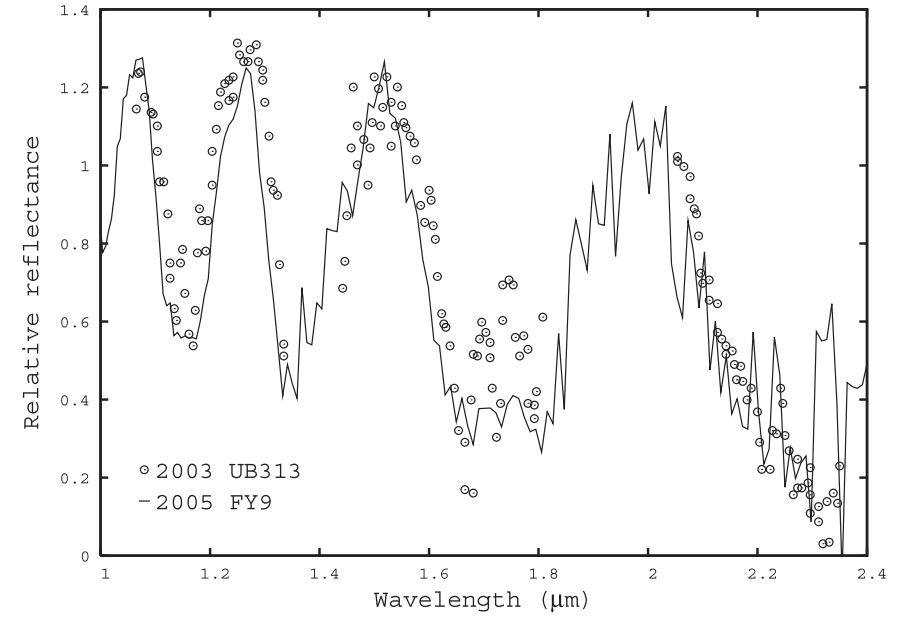

Fig. 3. Reflectance spectrum of $2005 \mathrm{FY}_{9}$ in the near-infrard range compared to the reflectance spectrum of TNO $2003 \mathrm{UB}_{313}$ (Brown et al. 2005d), normalized to match that of $2005 \mathrm{FY}_{9}$ around $1.6 \mu \mathrm{m}$. Notice that, apart from the different resolutions of the spectra, and within the $\mathrm{S} / \mathrm{N}$, both look very similar: the large methane ice absorption bands are observed with similar depths, indicating similar compositions.

Pluto's $\mathrm{CH}_{4}$ bands are seen to be partially shifted to shorter wavelengths relative to the wavelengths of pure methane ice absorption bands, indicating that at least some of the methane ice on Pluto's surface is diluted in $\mathrm{N}_{2}$ (Quirico et al. 1997; Schmitt et al. 1998; Douté et al. 1999). To measure the central wavelength and depth of the methane ice absorption bands is very difficult in our near-infrared spectrum owing to its very low resolution. But our visible spectrum has sufficient $\mathrm{S} / \mathrm{N}$ and resolution (see Fig. 2). Central wavelengths of the deeper methane ice bands in the visible spectrum of $2005 \mathrm{FY}_{9}$ are presented in Table 1 . The wavelengths are slightly shorter $(\sim 5 \AA)$ than those of pure methane ice, but within the error of our wavelength calibration $(\sim 7 \AA)$. A shift of only $\sim 17 \AA$ in the $0.89 \mu \mathrm{m}$ band is produced in a solid solution containing roughly $20 \%$ of methane dissolved in $\mathrm{N}_{2}$, as observed in Pluto. This shift results from 2 unresolved components: a longer wavelength pure $\mathrm{CH}_{4}$ absorption and a shorter wavelength diluted $\mathrm{CH}_{4}$ absorption. Smaller apparent shifts should result from higher $\mathrm{CH}_{4}$ concentrations. At present, we can not determine conclusively if some of the observed methane ice in $2005 \mathrm{FY}_{9}$ is diluted in $\mathrm{N}_{2}$, but its wavelength suggests it is mostly pure, or at least has a higher concentration than observed for Pluto's nearinfrared $\mathrm{CH}_{4}$ bands (e.g., Douté et al. 1999).

The prominent water ice absorption bands at 1.5 and $2.0 \mu \mathrm{m}$ are not apparent in the $2005 \mathrm{FY}_{9}$ spectrum, just as is true of Pluto (Brown 2002; Grundy \& Buie 2002). Water is essentially non-volatile at $40 \mathrm{~K}$. It must be abundant in the interior of TNOs from cosmochemical considerations, and was indeed detected in spectra of several of them. For $2005 \mathrm{FY}_{9}$, it would be difficult to detect a small fraction (a few percent) of water ice on its surface since near-infrared water ice absorption bands are masked by the strong methane ice bands in the nearinfrared. Much higher S/N spectra and detailed surface scattering models would be needed for a conclusive result, but our 
spectrum of $2005 \mathrm{FY}_{9}$ already excludes the presence of a high abundance of water ice exposed at its surface. We argue that, as is the case for Pluto, the surface of $2005 \mathrm{FY}_{9}$ has a mantle of volatile compounds blanketing water ice from the interior.

Considering the similarities in composition and size of Pluto and $2005 \mathrm{FY}_{9}$, and considering that Pluto has an atmosphere (e.g., Cruikshank \& Silvaggio 1980; Fink et al. 1980; Pasachoff et al. 2005), the possibility arises that $2005 \mathrm{FY}_{9}$ also has an atmosphere. According to Elliot \& Kern (2003), three conditions must be satisfied for a TNO-like object to have a bound atmosphere: (1) the body must have an inventory of volatiles on its surface that can sublimate; (2) the temperature must lie within the correct range - high enough for adequate vapour pressure, but not so high that the atmosphere would escape into space; (3) the body mass must be sufficient to retain an atmosphere. $2005 \mathrm{FY}_{9}$ seems to satisfy all three: volatiles are present at its surface, it orbits in a region slightly farther than Pluto (39-52 AU compared with 30-49 AU for Pluto) so its surface temperatures should be comparable or less than Pluto's, and its size is also similar to that of Pluto. We conclude that $2005 \mathrm{FY}_{9}$ is an excellent candidate to have an atmosphere similar to that of Pluto. As in the case of Pluto, the observation of an occultation of a star by $2005 \mathrm{FY}_{9}$ could detect this atmosphere.

Finally, Brown et al. 2005d presented a near infrared spectrum of $2003 \mathrm{UB}_{313}$ showing that it also has deep methane ice absorption bands. In fact, the spectra of $2005 \mathrm{FY}_{9}$ and $2003 \mathrm{UB}_{313}$ look very similar (3), but $2003 \mathrm{UB}_{313}$ is not as red in the visible (Brown et al. 2005d). The presence of frozen methane on the surfaces of Pluto, Triton, 2005 UB313 and $2005 \mathrm{FY}_{9}$ argues that the process suggested by Spencer et al. (1997) in which surface methane is replenished from the interior, may be ubiquitous in large trans-neptunian objects. $2005 \mathrm{FY}_{9}$ and $2003 \mathrm{UB}_{313}$ provide an exciting new laboratory for the study of processes considered for Pluto and Triton: volatile mixing and transport; atmospheric freeze-out and escape, ice chemistry, and nitrogen phase transitions.

\section{Conclusions}

We present a new $0.35-2.5 \mu \mathrm{m}$ spectrum of the TNO $2005 \mathrm{FY}_{9}$. The spectrum is very similar to that of Pluto, with a nearinfrared region dominated by prominent $\mathrm{CH}_{4}$ ice absorptions. At wavelengths $<0.6 \mu \mathrm{m}$ the spectrum is almost featureless and red. The $\mathrm{CH}_{4}$ ice bands in this new giant TNO are significantly stronger than those of Pluto, implying that methane could be even more abundant on its surface. The red color in the visible, almost the same as Pluto, suggests the presence of complex organics. The spectrum in the near-infrared is also very similar to that of TNO $2003 \mathrm{UB}_{313}$. The abundance of a volatile material such as methane at the probable surface temperature of $2005 \mathrm{FY}_{9}$, possibly accompanied by $\mathrm{N}_{2}$ and $\mathrm{CO}$, combined with a size comparable to that of Pluto, suggests that this Pluto-twin TNO is an excellent candidate to have a bound atmosphere.

Acknowledgements. Based on observations made with the Italian Telescopio Nazionale Galileo (TNG) operated on the island of
La Palma by the Fundación Galileo Galilei of the INAF (Istituto Nazionale di Astrofisica) at the Spanish Observatorio del Roque de los Muchachos of the Instituto de Astrofísica de Canarias J. Licandro thanks Luisa Lara-Lopez for her usefull suggestions. W. M. Grundy gratefully acknowledges support from NASA Planetary Geology \& Geophysics grant NNG04G172G. We also thanks the anonymous referee for his usefull comments to improve this paper.

\section{References}

Brown, M. 2002, Ann. Rev. Earth. Planet. Sci., 30, 307

Brown, M., \& Calvin, W. 2000, Science, 287, 107

Brown, R., Cruikshank, D., \& Pendleton, Y. 1999, ApJ, 519, L101

Brown, M. E., Trujillo, C. A., Rabinowitz, D., \& Marsden, B. G. 2005a, MPEC, 2005-O42

Brown, M. E., Trujillo, C. A., Rabinowitz, D., \& Marsden, B. 2005b, MPEC, 2005-O41

Brown, M. E., Trujillo, C., Rabinowitz, D., \& Marsden, B. 2005c, IAUC, 8577

Brown, M. E., Trujillo, C. A., \& Rabinowitz, D. 2005, ApJ, submitted Cruikshank, D. 1998, in Solar System Ices, ed. B. Schmitt et al. (Kluwer), 655

Cruikshank, D., \& Silvaggio, P. 1980, Icarus, 41, 96

Cruikshank, D., Pilcher, C., \& Morrison, D. 1976, Science, 194, 835

Cruikshank, D., Roush, T., Owem, T., et al. 1993, Science, 261, 742

Douté, S., Schmitt, B., Quirico, E., et al. 1999, Icarus, 142, 421

Edgeworth, K. 1949, MNRAS, 109, 600

Elliot, J., \& Kern, S. 2003, EM\&P, 92, 375

Fernández, J. 1980, MNRAS, 192, 481

Fink, U., Smith, B., Johnson, J., et al. 1980, Icarus, 44, 62

Fornasier, S., Dotto, E., Barucci, A., \& Barbieri, C. 2004, A\&A, 422, 43

Grundy, W. M., Schmitt, B., \& Quirico, E. 1993, Icarus, 105, 254

Grundy, W., \& Fink, U. 1996, Icarus, 124, 329

Grundy, W. M., \& Buie, M. W. 2001, Icarus, 153, 248

Grundy, W. M., \& Buie, M. W. 2002, Icarus, 157, 128

Grundy, W., Schmitt, B., \& Quirico, E. 2002, Icarus, 155, 486

Jewitt, D., \& Luu, J. 2004, Nature, 432, 731

Khare, B., Sagan, C., Arakawa, E., et al. 1984, Icarus, 60, 127

Kuiper G., 1951, in Astrophysics: A Topical Symposium, ed. J. A. Hynek (New York: McGraw Hill), 357

Landolt, A. 1992, AJ, 104, 340

Licandro, J., Oliva, E., \& Di Martino, M. 2001, A\&A, 373, L29

Licandro, J., Ghinassi, F., \& Testi, L. 2002, A\&A, 388, L9

Licandro, J., di Fabrizio, L., Pinilla-Alonso, N., et al. 2005, A\&A, submitted

Massey, P., Valdes, F., \& Barnes, J. 1992, in A User's Guide to Reducing Slit Spectra with IRAF,

http://iraf.noao.edu/iraf/ftp/iraf/docs/spect.ps.Z.

Ortiz, J. L., Bambery, R., Helin, E., et al. 2005, MPEC, 2005-O36

Owen, T., Roush, T., Cruikshank, D., et al. 1993, Science, 261, 745

Pasachoff, J. M., Souza, S. P., Babcock, B. A., et al. 2005, AJ, 129, 1718

Pinilla-Alonso, N., Licandro, J., \& Campins, H. 2004, A\&AS, DPS meeting, 36, 11.07

Quirico, E., \& Schmitt, B. 1997, Icarus, 127, 354

Schmitt, B., Quirico, E., Trotta, F., \& Grundy, W. M. 1998, in Solar System Ices, ed. B. Schmitt et al. (Kluwer Academic Publishers)

Spencer, J., Stansberry, J., Trafton, L., et al. 1997, in Pluto and Charon, ed. A. Stern, \& D. Tholen (Arizona Press), 435 